GA-A21520

\title{
OVERVIEW OF H-MODE STUDIES IN DIII-D
}

by

R.J. GROEBNER, S.L. ALLEN, ${ }^{(a)}$ D.R. BAKER, N.H. BROOKS, D.A. BUCHENAUER, K.H. BURRELL, T.N. CARLSTROM, M.S. CHU, S. CODA, ${ }^{(b)}$ J. CUTHBERTSON, ${ }^{(c)}$ E.J. DOYLE, ${ }^{(d)}$ T.E. EVANS, J.R. FERRON, D. FINKENTHAL, ${ }^{(e)}$ A.H. FUTCH, ${ }^{(a)}$ P. GOHIL, C.M. GREENFIELD, D.N. HILL, ${ }^{(a)}$ D.L. HILLIS, ${ }^{(f)}$

F.L. HINTON, J. HOGAN, $(f)$ C.L. HSIEH, A.W. HYATT, G.L. JACKSON, R. JONG, ${ }^{(a)}$ J. KIM, Y.B. KIM, C.C. KLEPPER, ${ }^{(f)}$ S. KONOSHIMA, ${ }^{(g)}$ R.J. LA HAYE, L.L. LAO, E.A. LAZARUS, $(f)$ A.W. LEONARD, S.I. LIPPMANN, M.A. MAHDAVI, R. MAINGI, $(f)$ W. MANDL, ${ }^{(h)}$ Y. MARTIN, ${ }^{(i)}$

R.A. MOYER, ${ }^{(d)}$ T.H. OSBORNE, W.A. PEEBLES, ${ }^{(d)}$ T.W. PETRIE, G.D. PORTER ${ }^{(a)}$

M.E. RENSINK, ${ }^{(a)}$ C.L. RETTIG, ${ }^{(d)}$ T. H. RHODES, ${ }^{(d)}$ M.J. SCHAFFER, D.P. SCHISSEL, R.P. SERAYDARIAN, R.T. SNIDER, G.M. STAEBLER, R.D. STAMBAUGH, H. St JOHN,

E.J. STRAIT, T.S. TAYLOR, D.M. THOMAS, S.J. THOMPSON, A.D. TURNBULL, M.R. WADE, $(f)$ J.G. WATKINS, ${ }^{(j)}$ W.P. WEST, R.D. WOOD, ${ }^{(a)}$ and D. WRÓBLEWSKI ${ }^{(a)}$

This is a preprint of a paper to be presented at the Fourth H-Mode Workshop, November 15-17, 1993, Naka, Japan, and to be printed in Plasma Physics and Controlled Fusion.

\section{Work supported by \\ U.S. Department of Energy}

Contract Nos. DE-AC03-89ER51114, DE-FG03-89ER51121, DE-FG03-86ER53225, DE-AC05-84OR21400, and DE-AC03-76DP00789

(a) Lawrence Livermore National Laboratory.

(b) Massachusetts Institute of Technology.

(c) Sandia National Laboratories.

(d) University of California at Los Angeles.

(e) University of California at Berkeley.

(f) Oak Ridge National Laboratory.

(g) Japan Atomic Energy Research Institute.

(h) Max-Planck Institute.

(i) Ecole Polytechnique Federale de Lausanne.

GENERAL ATOMICS PROJECT 3466 JANUARY 1994

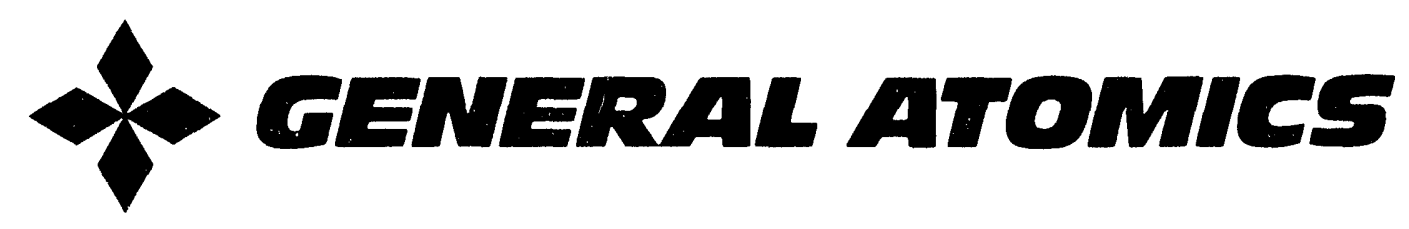




\section{Overview of $\mathrm{H}$-mode studies in DIII-D}

R.J. Groebner, S.L. Allen, ${ }^{a)}$ D.R. Baker, N.H. Brooks, D.A. Buchenauer, ${ }^{c)}$ K.H. Burrell, T.N. Carlstrom, M.S. Chu, S. Coda, ${ }^{\text {) }}$ J. Cuthbertson, ${ }^{c)}$ E.J. Doyle, d) T.E. Evans, J.R. Ferron, D. FinkenThal, ${ }^{e)}$ A.H. Futch, ${ }^{a)}$ P. Gohil, C.M. GReEnfield, D.N. Hill, ${ }^{a)}$ D.L. HiluIs, ${ }^{\text {f) }}$ F.L. Hinton, J. Hogan, ${ }^{f)}$ C.L. HsieH, A.W. HyaTT, G.L. JACKSON, R. JoNG, ${ }^{a)}$ J. KIM, Y.B. KIM, C.C. KLEPPER, ${ }^{f)}$ S. KonOSHIMA, $\left.{ }^{8}\right)$ R.J. LA HAYE, L.L. LAO, C.J. LASNIER, ${ }^{a)}$ E.A. Lazarus, ${ }^{f)}$ A.W. LeONard, S.I. LippManN, M.A. MaHdavi, R. MaINGI, W. MANDL, ${ }^{\text {h) }}$ Y. Martin, ${ }^{\text {i) }}$ R.A. Moyer, d) T.H. Osborne, W.A. Peebles, d) T.W. Petrie, G.D. Porter, M.E. Rensink, ${ }^{a)}$ C.L. RetTig, ${ }^{d)}$ T.H. Rhodes, ${ }^{d)}$ M.J. SchafFer, D.P. Schissel, R.P. Seraydarian, R.T. SNider, G.M. Staebler, R.D. Stambaugh, H. St John, E.J. Strait, T.S. Taylor, D.M. Thomas, S.J. Thompson, A.D. Turnbull, M.R. WAde, ${ }^{f}$ J.G. Watkins, ${ }^{c)}$ W.P. West, R.D. WOOD, ${ }^{a)}$ AND D. WRÓBLEWSKI ${ }^{a)}$

General Atomics, P.O. Box 85608, San Diego, California 92186-9784, U.S.A.

\section{(Received}

Abstract. A major portion of the DIII-D program includes studies of the L-H transition, of the VH-mode, of particle transport and control and of the powerhandling capability of a divertor. Significant progress has been made in all of these areas and the purpose of this paper is to summarize the major results obtained during the last two years. An increased understanding of the origin of improved confinement in $\mathrm{H}$-mode and in VH-mode discharges has been obtained, good impurity control has been achieved in several operating scenarios, studies of helium transport provide encouraging results from the point of view of reactor design, an actively pumped divertor chamber has controlled the density in $\mathrm{H}$-mode discharges and a radiative divertor is a promising technique for controlling the heat flux from the main plasma.

\section{Introduction}

Two major goals of the DIII-D program are obtaining an increased physics understanding of enhanced confinement modes in tokamaks and advancing the technology required to implement these confinement modes in a reactor. In pursuit of these goals, a significant portion of the DIII-D program is organized to study the physics of the formation of the $\mathrm{H}$-mode and VH-mode regimes of enhanced confinement, the transport and control of fuel and impurity species and the physics and control of the heat flux to the divertor plates. This paper will summarize major results obtained during the last two years from these areas of research.

\footnotetext{
a) Lawrence Livermore National Laboratory.

c) Sandia National Laboratories.

b) Massachusetts Institute of Technology.

d) University of California at Los Angeles.

e) University of California at Berkeley.

f) Oak Ridge National Laboratory.

g) Japan Atomic Energy Research Institute.

h) Max-Planck Institute.

i) Ecole Polytechnique Federale de Lausanne.
}

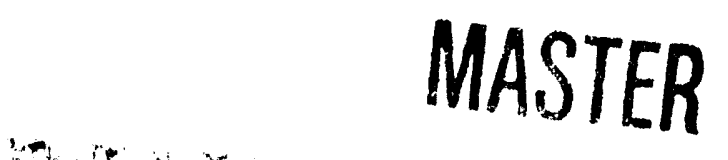


This paper is divided into four sections. Recent results of L-H transition studies will be presented in the first section. Direct measurements of changes in the electron density fluctuation level $\tilde{n}$ at the plasma edge at the transition are now available and support previous conclusions. Also, measurements of the terms of the lowest order radial force balance equation for the main ions provide new insights into transition physics. The second section presents results of VH-mode studies. Empirical iequirements to obtain this regime of confinement are examined. Observations from high confinement discharges suggest that sheared velocity fields are important in improving the confinement in both the core and edge. A survey of existing results regarding particle transport and control is presented in the third section. Present evidence indicates that the global particle confinement time $\tau_{\mathrm{p}}$ for the fuel ions is $\approx 1-4$ times the global energy confinement time $\tau_{\mathrm{E}}$. Transport studies of helium show no peaking relative to the electron density $n_{e}$ profile and that ELMs effectively purge helium from the plasma. Impurity radiation is at acceptable levels in ELMing $\mathrm{H}$-mode and $\mathrm{VH}$-mode discharges. Active density control has been obtained with a cryopump in the divertor chamber. Characterization and modelling of the scrape-off layer (SOL) and divertor regions are discussed in the fourth section. The goal of these studies is to predict and find ways to reduce the heat load to the divertor plates. A "radiative divertor" has been quite effective in reducing the peak divertor heat load. Conclusions and a discussion of future work are presented at the end of the paper.

\section{L-H transition}

A large body of evidence from many machines (Burrell et al., 1992; Groebner, 1993) shows that the formation of the transport barrier at the $\mathrm{L}-\mathrm{H}$ transition is consistent with the hypothesis that increased shear in the edge radial electric field $E_{\mathrm{r}}$ (actually, shear in the $\overrightarrow{\mathrm{E}}=(\overrightarrow{\mathrm{E}} \times \overrightarrow{\mathrm{B}}) / \mathrm{B}^{2}$ flow velocity) leads to a suppression of turbulence (Shaing et al., 1989; Biglari et al., 1990) and thus an improvement in confinement. Two new measurements provide additional evidence of a reduction of density fluctuations in the transport barrier, as has been previously inferred from measurements based on microwave reflectometry (Burrell etal., 1992; Doyle et al., 1993). A far infrared (FIR) collective scattering instrument shows that at the transition, reductions of the RMS level of electron density fluctuations $\tilde{n}$ of greater than $50 \%$ occur for frequencies in the electron diamagnetic drift direction (Rettig, 1993a; Rettig, 1993b). Previous studies have shown that the Doppler frequency shift of the fluctuations is dominated by the $\vec{E} \times \vec{B}$ velocity. Furthermore, numerous studies in DIII-D with coinjected neutral beams show that the only place in the plasma with a negative $E_{\mathrm{r}}$ is the transport barrier. Thus, it is concluded that the fluctuations in the electron diamagnetic drift direction are produced in the transport barrier. A complementary measurement has been made with a phase contrast interferometer system, which consists of a system of vertical chords passing through the edge of the plasma at the outer midplane. This instrument shows that for chords passing just inside the separatrix, the level of $\tilde{n}$ is reduced substantially at the L-H transition (Coda et al., 1993).

Measurements made with a Charge Exchange Spectroscopy (CER) system show that within a time resolution of $0.5 \mathrm{~ms}$, 㞰anges in $E_{\mathrm{r}}$ et the transition are coincident with the drop in the $\mathrm{D}_{\alpha}$ signal and are coincident with or slightly lead significant changes in pressure gradients (Burrell et al., 1992; Burrell et al., 1993a). The characteristic $E_{\mathrm{r}}$ "well" in $\mathrm{H}$-mode is initially very narrow, generally with a width of less than one $\mathrm{cm}$ and exists just inside the separatrix (Groebner et al., 1992; Gohil et al.; 1993). The width of the well is of the order of the ion poloidal gyroradius. In addition, the terms in the force balance equation have been measured for the main ions in a pure helium plasma, and the results show that by $3.5 \mathrm{~ms}$ after the transition the poloidal rotation velocity $v_{\theta}$ for the main ions is in the ion diamagnetic drift direction (Kim J. et al., 1993a; Kim J. et al., 1993b, Burrell et al., 1993a) and that the magnitude of $v_{\theta}$ is a significant fraction of the ion diamagnetic drift velocity. For the impurity species, $v_{\theta}$ is in the electron diamagnetic drift direction (Burrell etal., 1992; Groebner, 1993). The observed velocities for the impurities are in qualitative and moderate quantitative agreement with theories which predict that $v_{\theta}$ for the impurities is controlled by the pressure gradient of 
the main ions (Kim Y.B. et al., 1991; Valanju et al., 1992). Shortly after the transition, the pressure gradient term is the only term in the lowest order force balance equation for the main ions which contributes to a negative $E_{\mathrm{r}}$. At this time, measurements of higher order terms in the force balance equation are beyond the state of the art. It is conceivable that anomalous effects could make higher order terms important near the plasma edge. One consistency check which has been performed is that measurements of $E_{\mathrm{r}}$ based on impurity and main ions agree (Kim J. et al., 1993a; Kim J. et al., 1993b), even though the contributions of various terms in the lowest order force balance equation are not the same for all ions. Thus, if higher order terms are important, they must have the same magnitude for impurities and main ions.

The ultimate trigger of the spontaneous transition is not yet understood. The most compelling experimental picture of the trigger is that it is linked to edge temperature, and data from DIII-D are consistent with this picture. Measurements of edge $T_{\mathrm{i}}$ made with a spatial resolution of 3-6 mm and a temporal resolution of typically $2-10 \mathrm{~ms}$ show that $T_{\mathrm{i}}$ is rising continuously at the plasma edge during the L-mode phase (Groebner et al., 1992; Gohil et al., 1993). Furthermore, measurements of the main ions in a pure helium plasma show that the edge $\nu_{i} *$ is approximately one at the time of the transition (Burrell et al., 1992; Mandl et al., 1993). In these studies, the main ion density is measured directly, and thus $\nu_{i} *$ does not suffer from uncertainties in $n_{e}$ and $Z_{\text {eff }}$ as for measurements in which $\nu_{i} *$ is obtained indirectly.

Changes in the core profiles and density fluctuation start occurring after the formation of the transport barrier. These changes evolve over a time scale of tens of milliseconds (with $\tau_{\mathrm{E}}$ being of the order of $100 \mathrm{~ms}$ ). Time-resolved transport calculations show that the changes are consistent with a diffusive model of transport in which core heat transport coefficients (primarily outside the half-radius) decrease over tens of milliseconds (Kurki-Suonio et al., 1992; Kurki-Suonio et al., 1993). FIR scattering measurements show that $\tilde{n}$ decreases in the same region and with the same time scale as the transport coefficients are reduced (Philipona et al., 1993; Rettig et al., 1993b; Kurki-Suonio et al., 1993; Doyle et al., 1993). Possible reasons why microturbulence would be reduced in the core include a reduction of turbulence drive due to a flattening of the density gradient and an increase in the shear in $E_{\mathrm{r}}$ as the toroidal rotation $v_{\phi}$ steepens in the H-mode (Rettig, 1993b).

As is ubiquitously observed, ELM-free discharges either suffer a collapse due to radiated power or enter an ELMing phase. In DIII-D, the ELMing phase is obtained by injecting sufficient heating power. Although the origin of Type I and Type II ELMs is not understood, there is evidence that Type III ELMs may be due to resistive ballooning modes (Zohm et al., 1992). ELMing discharges suffer a confinement degradation of $\approx 15 \%$ (Schissel et al., 1992) relative to that expected from the JET/DIII-D scaling relationship for ELM-free H-mode discharges (Schissel et al., 1991). ELMing H-mode discharges also provide control of fuel and impurity densities, alleviate the radiation problem of ELM-free discharges and allow for steadystate operation.

\section{VH-mode}

Under conditions in which ELMs are suppressed and impurity sources and recycling of the fuel particles are adequately reduced, ELM-free $\mathrm{H}$-mode discharges routinely exhibit a transition to a regime of further enhanced confinement, called the VH-mode (Jackson et al., 1992a; Greenfield et al., 1992; Taylor et al., 1993; Osborne et al., 1993). Typical waveforms for a VH-mode discharge are shown in Fig. 1. VH-mode exhibits enhancements of the thermal energy confinement time $\tau_{\mathrm{E}}^{\text {thermal }}$ of 1.5-2.4 relative to the JET/DIII-D ELM-free H-mode values and of 3 to more than 4 relative to ITER-89P L-mode values (Yushmanov et al., 1990). VH-mode discharges in DIII-D have provided confinement times $\tau_{\mathrm{E}}^{\text {thermal }}$ of over $400 \mathrm{~ms}$ and a value of the triple fusion product $n_{D}(0) T_{i}(0) \tau_{E}^{\text {thermal }} \approx 4 \times 10^{20} \mathrm{~m}^{-3}-\mathrm{keV}-\mathrm{s}$ (Greenfield et al., 1993), where $n_{D}(0)$ and $T_{i}(0)$ are the deuteron density and ion temperature at the magnetic axis. Impurity and particle control was initially obtained with boronization of the vessel when the wall was partially bare inconel and partially covered with graphite tiles. Prior to the experimental 
campaign of 1993, all graphite tiles in the vessel were removed, cleaned of metallic deposits and reinstalled. In addition, new graphite tiles were installed to provide complete coverage of the vessel interior with graphite. With this full graphite coverage, the VH-mode has also been achieved without boronization.

The confinement improvement of the VH-mode is much more of a core phenomenon than the L-H transition. The steep gradients of the transport barrier extend substantially further into the discharge (Jackson et al., 1992a). The thermal diffusivities obtained from power balance analysis decrease substantially in the region where the gradients steepen (Greenfield et al., 1992). During the duration of the VH-mode, the ratio of radiated power $P_{\text {rad }}$ to injected power $P_{\text {inj }}$ remains low, typically less than 0.3 (Fig. 1), even though there are no ELMs (Jackson et al., 1992a). VH-mode discharges obtained so far are normally terminated by a global loss of energy with the discharge rapidly reverting to an ELMing $\mathrm{H}$-mode discharge. The termination is initiated by a low $n$ instability (typically $n=3-5$ ) localized to the edge region (Osborne et al., 1993). Experimental evidence and stability calculations suggest that this instability is an ideal kink (Strait et al., 1993). The termination appears to be a manifestation of a beta limit due to the rising bootstrap current density and pressure gradient near the plasma edge.

A number of hypotheses for the origin of the good confinement of the VH-mode have been examined and two important factors have emerged. First, it appears important that the plasma edge be stable against ELMs (Osborne et al., 1993; Greenfield et al., 1993). In DIII-D, this condition is generally obtained by operating discharges with high triangularity $(\delta \approx 0.6-0.9)$ which have improved access to the second stable region for ideal ballooning modes. Although VH-mode discharges have been obtained in low triangularity discharges $(\delta \approx$ 0.3-0.6), confinement enhancements have not been as good as in high triangularity discharges, and the reason may be that ELMs occur in the low trangularity discharges before the highest confinement times can be achieved. In addition, there is good correlation between an increase in the volume of plasma with highly sheared toroidal rotation velocity $v_{\phi}$ profiles and the region of improved confinement (Osborne etal., 1993; Greenfield et al., 1993). For these discharges, the $v_{\phi}$ profile in the core is steepening with time after the application of auxiliary heating and a continuous reduction of thermal transport coefficients in the region of increased shear is observed (Osborne et al., 1993; Greenfield et al., 1993). In addition, for discharges in which $\tau_{E} / \tau_{\text {JET } / D I I-D}$ exceeds 1.5, a marked "spin-up" of $v_{\phi}$ is observed (Osborne et al., 1993; Burrell et al., 1993a; Burrell et al., 1993b; Fig. 1). In the same region of the plasma where the spinup occurs, an instability observed with an FIR scattering system is quenched (Osborne et al., 1993; Burrell et al., 1993b; Rettig et al., 1993c). This quenching leads to a noticeable increase in the local gradient of $T_{\mathrm{I}}$ and to an increase in the rate of rise of $\tau_{\mathrm{E}}$. Increasing shear in $v_{\phi}$ implies increasing shear in $E_{\varepsilon}$ and thus stabilization due to shear in the $v_{E}$ velocity may be important. Recently, it has been pointed out that shear in $v_{\|}\left(\simeq v_{\phi}\right)$ can also stabilize turbulence and may be operative in the DIII-D VH-mode (Shaing et al., 1993; Staebler et al., 1993). In these discharges, toroidal rotation is produced by uni-directional neutral beam injection. Supporting evidence for the shear hypothesis is provided by experiments in which an externally applied field error has been used to reduce the shear in $v_{\phi}$ with a corresponding decrease in $\tau_{E}$ (Lahaye et al., 1993).

The origin of the VH-mode is obviously linked to wall conditions, but the reason for the link is not clearly understood. Boronization markedly reduced the level of impurities in the plasma. In Ohmic discharges, oxygen levels were reduced by a factor of about 6 , carbon levels by about 2 and nickel levels by a factor of 10-15 with more variable but significant reductions in auxiliary-heated discharges (Lippmann et al., 1992). Likewise, the all-graphite wall has reduced the level of impurities to very low levels. It is also crucial to have very low recycling of the working gas in order to obtain VH-mode. The maximum $\tau_{\mathrm{E}}$ value achieved varies inversely with the recycling level (Jackson et al., 1992b). Well-conditioned walls, which have a low inventory of neutral deuterium gas, are obtained by a careful program of baking, tokamak discharges and helium glow discharge cleaning before every plasma discharge (Jackson et al., 1990). It is possible that the empirical requirements to obtain low recycling and to avoid 

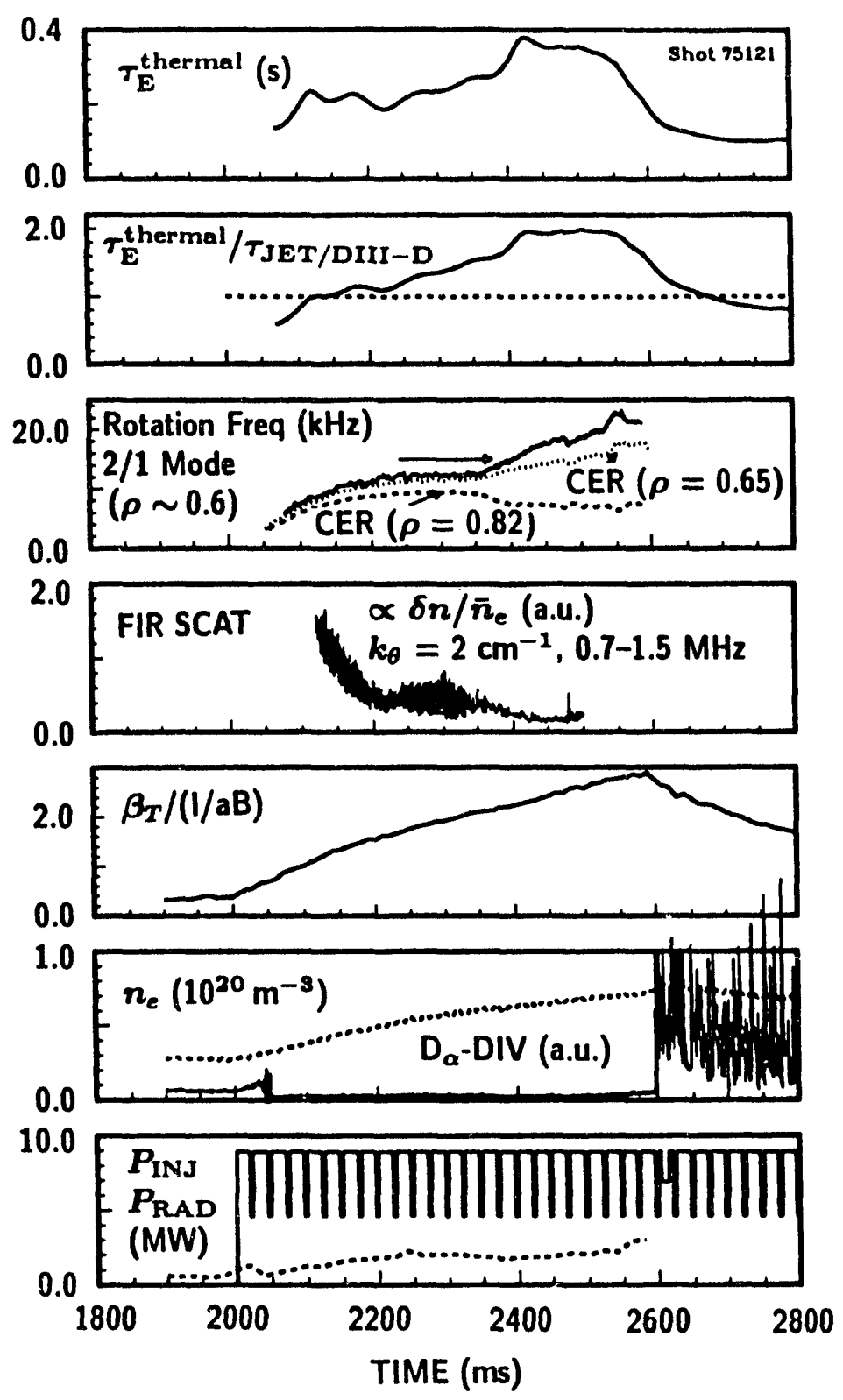

Figure 1. Typical waveforms for a VH-mode discharge. (a) Thermal energy confinement time increases during ELM-free phase and increases rapidly shortly after spinup, which starts at about $2350 \mathrm{~ms}$; (b) $\tau_{\mathrm{E}}^{\text {thermal }}$ reaches twice the value expected from JET/DIII-D scaling; (c) toroidal rotation frequency from two CER chords straddling the region of maximum spin-up and from $2 / 1$ MHD mode. ( $\rho$ is normalized toroidal flux.) (d) bursts of density fluctuations, observed by FIR scattering in region of plasma in which spin-up occurs, are quenched at time of spin-up (With the time resolution of this figure, the bursts of fluctuations appear as hash on the signal; when observed with finer time resolution, it is clear that the hash is due to discrete bursts of fluctuations.); (e) normalized toroidal beta $\left(\beta_{T} /\left(I_{P} / a B_{T}\right)\right.$ where $\beta_{T}$ is toroidal beta, $I_{P}$ is plasma current, $a$ is the minor cross section and $B_{T}$ is the toroidal magnetic field) reaches value of 2.9 at termination of VH-mode; (f) electron density and divertor $\mathrm{D}_{\alpha} ;(\mathrm{g})$ radiated power $P_{\text {rad }}$ is less than $30 \%$ of injected heating power $P_{\mathrm{inj}}$. 
ELMs are actually the same requirement. It is known empirically that higher recycling leads to ELMs occurring earlier in an $\mathrm{H}$-mode discharge and thus an indirect effect of improved wall conditions may be to increase the length of the ELM-free period.

\section{Particle transport and control}

The transport and control of fuel ions, alpha particles and impurities are topics of great importance for reactor design but are relatively poorly understood. The concentration of a given species in the plasma is determined by the source strength for production of the species, the efficiency for penetration into the plasma and the transport of the particles in the plasma (Lippmann et al., 1992). The interplay between these three variables is important to understand particle transport in DIII-D. For example, low impurity levels in $\mathrm{H}$-mode and VH-mode discharges are partly the result of the reduction of the source strength for both low-z and metallic impurities by boronization or the use of an all-graphite wall. In addition, helium glow discharge conditioning reduces the wall source for neutrals of the fuel gas.

The marked increase in $n_{e}$ after the L-H transition has often been interpreted as indicating that $\tau_{\mathrm{p}}$ increases much more than $\tau_{\mathrm{E}}$ from $\mathrm{L}$-mode to $\mathrm{H}$-mode. (The global particle confinement time for the fuel ions $\tau_{\mathrm{p}}$ is obtained from $\mathrm{dN} / \mathrm{dt}=\mathrm{S}-\mathrm{N} / \tau_{\mathrm{p}}$ where $\mathrm{N}$ is the total number of deuterons in the plasma and $S$ is the source rate in the plasma for deuterons.) However, detailed calculations of the neutral source, based on the Monte Carlo code DEGAS and appropriate experimental measurements, show that reduced screening is an important reason for the large increase in density (Rensink et al., 1993). The probability for recycling deuterium neutrals to penetrate into the plasma is higher in $\mathrm{H}$-mode than in $\mathrm{L}$-mode due to the thinner SOL of the $\mathrm{H}$-mode. Transport calculations using the neutral source obtained by this technique show that the ratio of $\tau_{\mathrm{p}}$ to $\tau_{\mathrm{E}}$ for the deuterons is the same in $\mathrm{L}$-mode and $\mathrm{H}$-mode. $\tau_{\mathrm{p}}$ and $\tau_{\mathrm{E}}$ both increase by a factor of two with $\tau_{p}$ found to be a factor of about four larger than $\tau_{E}$ in $\mathrm{L}$-mode and H-mode (Rensink et al., 1993). An upper limit for $\tau_{\mathrm{p}}$ in VH-mode discharges has been inferred from measurements of $\tau_{\mathrm{p}}^{*}=\tau_{\mathrm{p}} /(1-R)$ with $R$ being the recycling coefficient. Analysis of the time rate of change of $n_{e}$ shows that $\tau_{p}^{*}$ is $\approx 1$ times $\tau_{E}$ (Jackson et al., 1992b). Since $R$ is less than one for these discharges, it can be concluded that $\tau_{\mathrm{p}}$ is no greater than $\tau_{\mathrm{p}}^{*}$ and therefore no greater than $\tau_{\mathrm{E}}$. The difference in the ratio of $\tau_{\mathrm{p}} / \tau_{\mathrm{E}}$ obtained from these two measurements may be due to uncertainties in these measurements or may reflect differences of particle confinement in $\mathrm{VH}$-mode relative to $\mathrm{L}$-mode and $\mathrm{H}$-mode.

Studies are being made of the transport of fully ionized helium at concentrations of several percent of $n_{e}$. The $\mathrm{He}^{++}$profile is found to have the same shape as the $n_{e}$ profile for all standard regimes of operation, including L-mode, ELM-free H-mode and ELMing $\mathrm{H}$-mode discharges (Finkenthal et al., 1992; Hillis et al., 1993; Wade et al., 1993). That is, no peaking of the $\mathrm{He}^{++}$ profile is observed relative to the $n_{e}$ profile. ELMs tend to purge the plasma of helium. During each ELM, a significant amount of helium is lost from the plasma and simultaneously there is an increase of helium in the divertor chamber, as deduced from the sharp rise in an $\mathrm{He}^{+}$ line (Hillis et al., 1993; Wade et al., 1993). Transport modelling shows that the $\mathrm{He}^{++}$diffusion coefficient in the core plasma decreases relative to the global energy confinement time as the ELM frequency increases (Wade et al., 1993).

The confinement of low and medium $\mathrm{Z}$ impurities is not as well characterized as for helium, but it is clear that ELMing H-mode and VH-mode discharges can be run without serious impurity problems. VH-mode discharges have volume-averaged $Z_{\text {eft }}$ values which are typically no greater than two. Despite having no ELMs, these discharges show no increase in radiation from nickel ions and exhibit values of $P_{\mathrm{rad}} / P_{\text {inj }}$ of $\leq 30 \%$ or less which remain steady during the duration of the discharge, with the possible exception of the last $100 \mathrm{~ms}$ before the termination event. For reasons not understood, VH-mode discharges typically have substantial SOLs with $n_{e} \approx 1-2 \times 10^{18} \mathrm{~m}^{-3}$ and $T_{e} \leq 10 \mathrm{eV}$ in the SOL (Lippmann et al., 1992). Screening by the SOL plasma coupled with the reduced impurity source due to boronization or all-graphite walls may be the reason why $P_{\text {rad }}$ remains low in VH-mode discharges. 
A divertor plate (ADP) which can be biased relative to the vacuum chamber has been available since 1990 . With biasing techniques, it has been possible to substantially increase the pressure in the baffled chamber (Schaffer et al., 1993a; Schaffer et al., 1993b). Recently, a cryopump has been installed under the divertor baffle plate (Schaubel et al., 1993) to provide active control of the plasma density. The cryopump is very effective in pumping neutrals which arrive in the divertor chamber and has been used to reduce the density by up to a factor of two in ELMing H-mode discharges (Mahdavi et al., 1993, Schaffer, 1993b).

\section{Divertor heat flux studies}

Reactor studies and the ITER conceptual design activity have led to great concerns as to whether divertor plates would have acceptable lifetimes in the face of the expected heat flux. To study this issue and with the ultimate goal of building a possible ITER-prototype divertor chamber, the DIII-D program has a major effort to characterize and model the divertor and to search for acceptable solutions to the divertor heat load problem. Characterization of the physics of the SOL and divertor region (Hill et al., 1993) is accomplished with a comprehensive and growing complement of diagnostics. Among the parameters measured are the temperatures and densities in the SOL at the midplane and at the divertor plate, the divertor heat flux and the recycling $D_{\alpha}$ emission and the radiated power in the divertor region. Numerical simulation codes are used to model the radial and parallel transport in the scrape-off layer and to make predictions of the heat flux at the divertor plate (Porter et al., 1992). Comparisons with measured heat fluxes show that the models calculate the correct footprint for the heat deposition and closely obtain the magnitude of the heat flux (Porter et al., 1993). These studies uncover additional important physics which is subsequently included in the modeling.

Experimentally, it has been found that with an appropriate gas puff into the divertor chamber, much of the heat entering the divertor $r$ gion can be radiated to the walls and the peak heat load to the divertor plate has been reduced by up to a factor of five (Fig. 2) (Petrie et al., 1992; Petrie et al., 1993). Operated in this way, the divertor is referred to as a "radiative" divertor. These results have been maintained for the duration of the H-phase (roughly $2 \mathrm{~s}$ ) in ELMing H-mode discharges with $\tau_{\mathrm{E}}$ at $2 \mathrm{x}$ the ITER-89P value (Petrie et al., 1993). A variety of ideas are being tested to see if impurities can be used to enhance the radiation in the divertor with little effect on the main plasma. Experimental and modeling efforts will continue to predict the heat flux deposited on the divertor plates and this effort will be used in the design of a new radiative divertor for DIII-D. The goal of this design is to reduce the peak divertor heat flux by an order of magnitude relative to conventional divertors, and installation of this divertor chamber in DIII-D is planned for 1995 (Simonen, 1992).

In discharges with Type I ("Giant") ELMs, it is found that ELMs account for more than $50 \%$ of the particle loss from the main plasma but for less than $20 \%$ of the energy losses (Hill et al., 1992). These findings are consistent with previous results that ELMs are quite effective at controlling the density rise while reducing the energy confinement time by less than $20 \%$. While it is true that an ELM transiently increases and modifies the heat deposition to the divertor plate, the temperature profile on the divertor plate at the end of beam heating has the shape of the steady state heat deposition (Hill et al., 1992). Thus, steady state losses dominate the total energy delivered to the plates.

\section{Conclusions and future work}

The DIII-D program is directed towards developing tokamak concepts which lead to a commercially attractive demonstration fusion reactor. Towards this end, a major portion of the program is dedicated to studies of the enhanced confinement regimes of $\mathrm{H}$-mode and VH-mode and to work in the areas of particle control and control of the divertor heat load. An overview of these studies has been presented in this paper. 


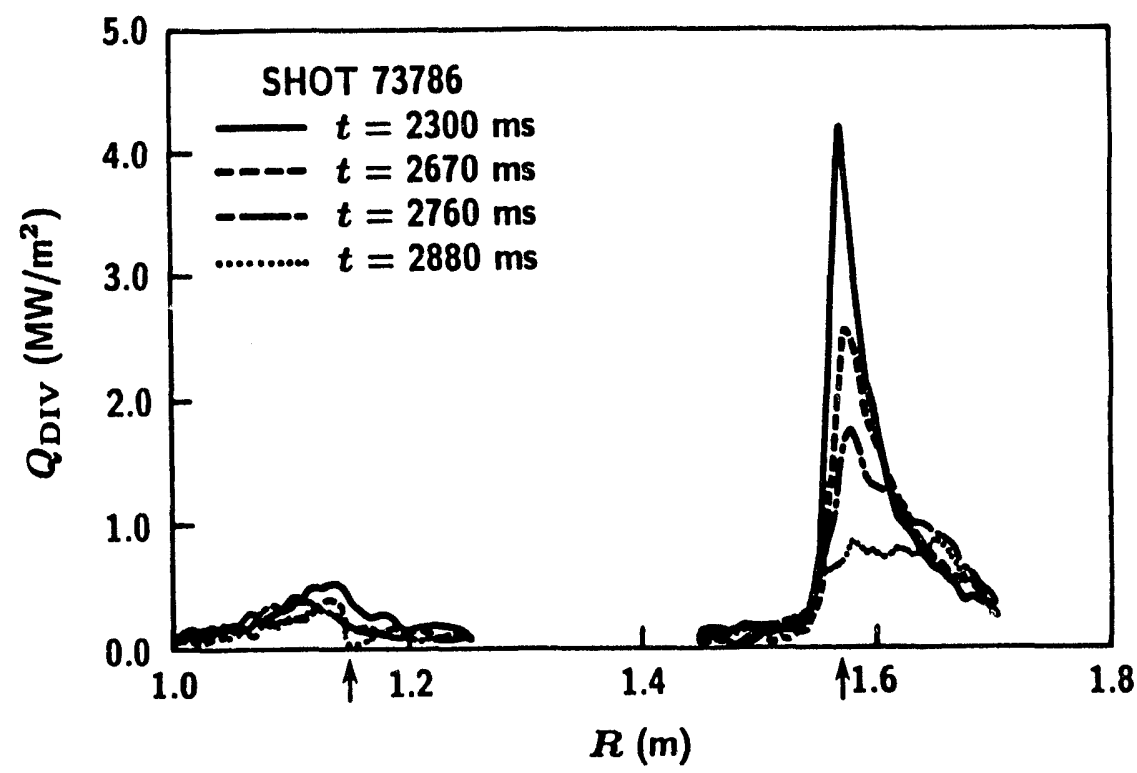

Figure 2. Radial profile of heat flux to inner and outer strike points of divertor plate before and during deuterium gas puff. Gas puff started at $2300 \mathrm{~ms}$ and by $2880 \mathrm{~ms}$ the peak heat flux to outer strike point $(R=1.580 \mathrm{~m})$ was reduced by a factor of $4-5$.

The hypothesis that sheared velocity fields can reduce turbulence is an attractive candidate for explaining the detailed observations of the formation of the transport barrier at the $\mathrm{L}-\mathrm{H}$ transition and of the core confinement improvement in VH-mode. Large shear in the $v_{\mathrm{E}}$ velocity is observed in the transport barrier of the $\mathrm{H}$-mode and large shear in $v_{\mathrm{E}}$ and in $v_{\|}$due to large shear in $v_{\phi}$ are observed in the VH-mode in the region where the core confinement improves. A continuing area of study is the origin of $E_{\mathrm{r}}$ at the plasma edge. In addition, a more thorough characterization of edge fluctuations in $\mathrm{L}$-mode and in $\mathrm{H}$-mode will be obtained from an increasing complement of fluctuations diagnostics.

ELMing H-mode discharges exhibit confinement times which are reduced by about $15 \%$ relative to those expected from JET/DIII-D ELM-free scaling, but the values are well above I-mode levels. These discharges provide control of electron density and impurity radiation. Previous long pulse $\mathrm{H}$-mode studies have indicated a need for greater control of the neutral density, and that control has now been obtained with an actively-pumped divertor chamber. The heat load to the divertor plates is dominated by steady state losses rather than by ELMs, possibly mitigating concerns that the transient heat load on the divertor plates due to ELMs might be a major problem for future divertor designs. ELMs purge helium from the plasma and provide a scenario which appears to be attractive for helium control and exhaust in a reactor.

Improved wall conditions leading to reduced impurity levels and low levels of recycling of the fuel gas have allowed ELM-free $\mathrm{H}$-mode discharges to access a further enhanced mode of confinement called the VH-mode. By operating at low values of $P_{\text {rad }} / P_{\text {inj }}$, the VH-mode demonstrates that good control of the impurity source is possible. In addition, screening by the scrape-off layer appears to play a significant role in keeping impurities out of VH-mode discharges. Due to its high confinement time and operation at high normalized beta, the VH-mode discharge offers many properties which are desireable for a reactor. Thus, future studies will be directed towards obtaining a deeper understanding of the termination event, which appears to be a beta limit, and towards schemes to control the edge current density or pressure gradient with the goal of lengthening the duration of the VH-phase.

Two studies of particle confinement for the main ions have been performed for DIII-D. The result of one study, based on detailed modeling of the source for the deuterons, is that the ratio of the global particle confinement time $\tau_{\mathrm{p}}$ for the fuel ions to $\tau_{\mathrm{E}}$ is approximately the same in L-mode and ELM-free H-mode discharges and that $\tau_{\mathrm{p}}$ is $\approx 4$ times $\tau_{\mathrm{E}}$. This work also 
shows that thinning of the SOL after the transition singificantly increases the probability for neutrals to penetrate into the discharge and contributes to the large rise in density observed in the $\mathrm{H}$-mode. In another study, measurements of $\tau_{\mathrm{p}}^{*}$ have been used to provide an upper limit for an estimate of $\tau_{\mathrm{p}}$ in $\mathrm{VH}$-mode discharges. The results indicate that $\tau_{\mathrm{p}}$ is comparable to $\tau_{\mathrm{E}}$ in these VH-mode discharges. The differences between these two studies may reflect difficulties in measuring particle transport or may indicate that the ratio $\tau_{\mathrm{p}} / \tau_{\mathrm{E}}$ is reduced in $\mathrm{VH}$-mode relative to standard $\mathrm{H}$-mode discharges.

Control of the fuel particles is important in many operating regimes and effective particle control has been obtained by conditioning the vessel walls and with a cryopump in the divertor chamber. For concentrations of $\mathrm{He}^{++}$of a few percent of $n_{e}$, there is no peaking of the $\mathrm{He}^{++}$ relative to $n_{e}$. Studies of particle transport, including helium, will be expanded in DIII-D. In addition, the ability of divertor cryopumping to control the density in various parameter regimes will be studied more extensively.

\section{Acknowledgments}

The results presented here are the result of the dedication and hard work of the entire DIII-D staff, including the technician, engineering, operations, computer and physics groups. The divertor program is a collaborative effort between GA, LLNL, ORNL, SNL and UCLA with participation from several other institutions. This work was supported by the U.S. Department of Energy under Contract Nos. DE-AC03-89ER51114, DE-FG03-89ER51121, DE-FG0386ER53225, DE-AC05-84OR21400, and DE-AC03-76DP00789.

\section{References}

Biglari, H., P. H. Diamond, and P. W. Terry (1990). Phys. Fluids B2, 1.

Burrell, K. H. (1993a). To be published in Phys. Fluids.

Burrell, K. H., T. H. Osborne, R. J. Groebner and C. L. Rettig, (1993b). Proc. 20th European Conf. on Controlled Fusion and Plasma Physics, Lisboa, Vol. 17C, p. 27.

Burrell, K. H., et al., (1992). Plasma Phys. Contr. Fusion 34, 1859.

Coda, S., M. Porkolab, K. H. Burrell, and T. N. Carlstrom, (1993). Proc. 20th European Conf. on Controlled Fusion and Plasma Physics, Lisboa, Vol. 17C, p. 1179.

Doyle, E. J., etal., (1993). Plasma Physics and Controlled Nuclear Fusion Research 1992 (LAEA, Vienna, 1993), Vol. 1, p. 235.

Finkenthal, D. F., et al., (1992). Proc. 19th European Conf. on Controlled Fusion and Plasma Physics, Innsbruck, Vol. 16C, p. 275.

Gohil, P., K. H. Burrell, E. J. Doyle, and R. J. Groebner, (1993). Submitted for publication in Nucl. Fusion.

Greenfield, C. M., et al., (1992). Proc. 19th European Conf. on Controlled Fusion and Plasma Physics, Innsbruck, Vol. 16C, p. 11.

Greenfield, C. M., et al., (1993a). Plasma Phys. Contr. Fusion, to be published.

Groebner, R. J., K. H. Burrell, P. Gohil, Y. B. Kim, and R. P. Seraydarian, (1992). Proc. 19th

European Conf. on Controlled Fusion and Plasma Physics, Innsbruck, Vol. 16C, p. 183.

Groebner, R. J., (1993). Phys. Fluids B5, 2343.

Hill, D. N., et al., (1992). J. Nucl. Mater. 196-198, 204.

Hill, D. N., et al., (1993). Proc. 20th European Conf. on Controlled Fusion and Plasma Physics, Lisboa, Vol. 17C, p. 643.

Hillis, D. L., etal., (1993). Plasma Physics and Controlled Nuclear Fusion Research 1992 (IAEA, Vienna, 1993), Vol. 1, p. 577.

Jackson, G. L., T. S. Taylor, and P. L. Taylor, (1990). Nucl. Fusion 30, 2305.

Jackson, G. L., et al., (1992a). Phys. Fluids B4, 2181.

Jackson, G. L., et al.(1992b). Bull. Amer. Phys. Soc. 37, 1509. 
Lippmann, S. I., T. E. Evans, G. L. Jackson and W. P. West, (1992). J. Nucl. Mater. 196-198, 498.

Kim, J., K. H. Burrell, P. Gohil, R. J. Groebner, Y. B. Kim, H. E. St. John, R. P. Seraydarian, and M. R. Wade, (1993a). Phys. Rev. Lett., submitted for publication.

Kim, J. et al., (1993b). To be published in Proceedings of 1993 IAEA Technical Committee Meeting on $\mathrm{H}$-mode Physics, Plasma Phys. and Contr. Fusion.

Kim, Y. B., P. H. Diamond, and R. J. Groebner, (1991). Phys. Fluids B3, 2050.

Kurki-Suonio, T. K., R. J. Groebner, and K. H. Burrell, (1992). Nucl. Fusion 32, 133.

Kurki-Suonio, T.K., K.H. Burrell, R.J. Groebner, R. Philipona and C.L. Rettig, (1993). Nucl. Fusion 33, 301.

La Haye, R. J. et al., (1993). To be published in Proceedings of Varenna Workshop on Local Thansport Studies in Fusion Plasmas

Mahdavi, M. A., et al., (1993). Proc. 20th European Conf. on Controlled Fusion and Plasma Physics, Lisboa, Vol. 17C, p. 647.

Mandl, W., (1993). To be published.

Osborne, T. H., et al., (1993). General Atomics Report GA-A21182, submitted to Nucl. Fusion.

Petrie, T. W., and the DIII-D Physics Group (1993). Bull. Amer. Phys. Soc. 38, 2071 and to be published.

Petrie, T. W., et al., (1992). J. Nucl. Mat. 196-198, 848.

Philipona, R., et al., (1993). Phys. Fluids B5, 87.

Porter, G. D., M. E. Rensink, T. D. Rognlien, R. A. Jong, and D. N. Hill, (1992). Proc. 19th European Conf. on Controlled Fusion and Plasma Physics, Innsbruck, Vol. 16C, p. 751.

Porter, G. D., M. E. Rensink, T. D. Rognlien, (1993). Bull. Amer. Phys. Soc. 38, 1936.

Rensink, M. E., S. L. Allen, A. H. Futch, D. N. Hill, M. A. Mahdavi, and G. D. Porter, (1993). Phys. Fluids B5, 2165.

Rettig, C. L., et al., (1993a). Nucl. Fusion 33, 643.

Rettig, C. L., et al., (1993b). Phys. Fluids B5, 2428.

Rettig, C. L., etal., (1993c). To be published in Proc. of 1993 IAEA Technical committee Meeting on H-mode Physics, Plasma Phys. and Contr. Fusion.

Schaffer, M. J., etal., (1993a). Plasma Physics and Controlled Nuclear Fusion Research 1992 (LAEA, Vienna, 1993), Vol. 1, p. 299.

Schaffer, M. J., et al., (1993b). to be published in Proc. of 9th IAEA Workshop on Stellarators, Garching, 1993

Schaubel, K. M., etal., (1993). to be published in Advances in Cryogenic Engineering 39.

Schissel, D. P., et al., (1991). Nucl. Fusion 31, 73.

Shaing, K. C., and E. C. Crume, Jr., (1989). Phys. Rev. Lett. 63, 2369.

Shaing, K. C., (1993). Phys. Fluids, to be published.

Simonen, T. C., (1992). J. of Fusion Energy 11, 79.

Staebler, G. M., et al., (1993). Phys. Fluids, submitted for publication.

Strait, E. J., etal., (1993). Proc. 20th European Conf. on Controlled Fusion and Plasma Physics, Lisboa, Vol. 17C, p. 211.

Taylor, T. S., etal., (1993). Plasma Physics and Controlled Nuclear Fusion Research 1992 (LAEA, Vienna, 1993), Vol. 1, p. 167.

Valanju, P. M., M. D. Calvin, R. D. Hazeltine, and E. R. Solano, (1992). Phys. Fluids B4, 2675.

Wade, M. R., etal., (1993). Proc. 20th European Conf. on Controlled Fusion and Plasma Physics, Lisboa, Vol. 17C, p. 63.

Yushmanov, P. N., et al., (1990). Nucl. Fusion 30, 1999.

Zohm, H., et al., (1992). Proc. 19th European Conf. on Controlled Fusion and Plasma Physics, Innsbruck, Vol. 16C, p. 243. 

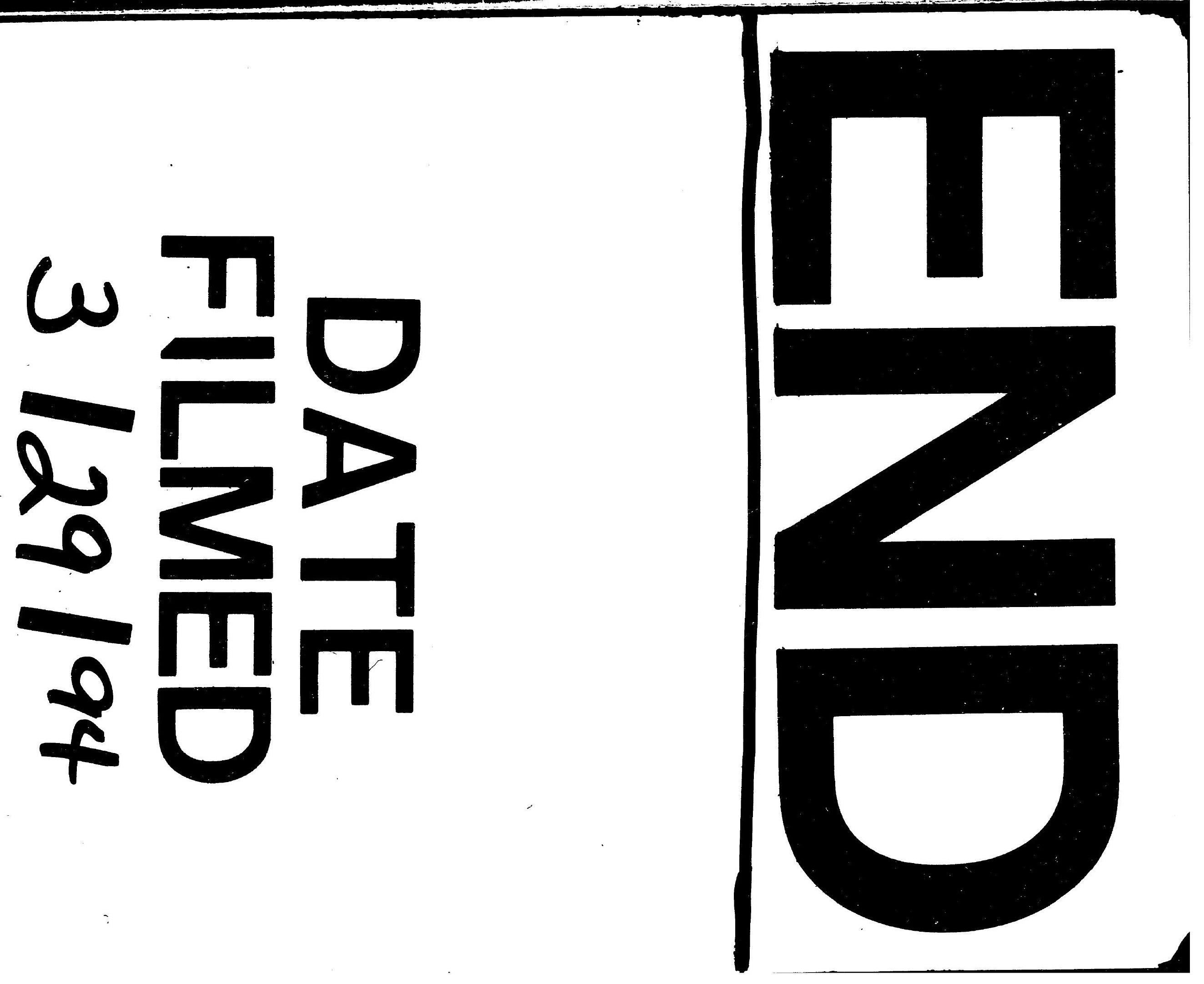